\title{
BROTACIÓN DEL MANZANO (Malus domestica BORKH. CV. 'ANNA') EN RESPUESTA A LA ÉPOCA DE DEFOLIACIÓN
}

\author{
BUDBREAK OF APPLE TREES \\ (Malus domestica BORKH. CV. 'ANNA') \\ IN RESPONSE TO DEFOLIATION TIMING
}

\author{
Fánor Casierra-Posada ${ }^{1}$ \\ Carlos A. Sepúlveda ${ }^{2}$ \\ Óscar E. Aguilar-Avendaño ${ }^{3}$
}

\begin{abstract}
RESUMEN
A pesar que las condiciones en zonas tropicales han sido establecidas para el manzano 'Anna', poco se conoce acerca de los eventos bioquímicos y fisiológicos que marcan el final de los requerimientos de horas frío y el comienzo de la brotación. Bajo las condiciones climáticas de Colombia, las yemas florales de manzano brotan en cualquier época, como respuesta a la caída de hojas después de la cosecha, a la poda o a la ayuda con inductores químicos. El presente trabajo, se llevó a cabo en Paipa, Colombia, para evaluar la respuesta de árboles de manzano cv 'Anna' a diferentes épocas de defoliación. Se defoliaron árboles una semana antes, durante y de una a cuatro semanas posteriores a la cosecha. Se utilizaron plantas control, sin defoliación, permaneciendo las hojas en sus ramas durante el periodo
\end{abstract}

\footnotetext{
${ }^{1}$ Profesor asociado en la Facultad de Ciencias Agropecuarias de la Universidad Pedagógica y Tecnológica de Colombia, grupo de investigación Ecofisiología Vegetal, Apartado Aéreo 661, Tunja - Boyacá, Colombia. E-mail: fanor. casierra@uptc.edu.co [Autor para correspondencia]

${ }^{2}$ Ingeniero Agrónomo, Coordinador Técnico Comercial de Barpen S.A para Cundinamarca y Boyacá. E-mail: csepulveda@barpen.com

${ }^{3}$ Estudiante Ingeniería Agronómica, Universidad Pedagógica y Tecnológica de Colombia, Tunja - Boyacá. E-mail: oskareduardo1@hotmail.com
}

del experimento, hasta su caída natural. Las variables medidas fueron: contenido de carbono y nitrógeno, la relación $\mathrm{C} / \mathrm{N}$ y el peso seco en las yemas florales al momento de la defoliación y de la brotación y los días transcurridos de defoliación a brotación. La remoción de hojas redujo el tiempo de defoliación a brotación; el momento de defoliación afectó positivamente el porcentaje de brotación y el peso seco de las yemas florales, no obstante, los contenidos de carbono y nitrógeno, permanecieron inalterados. Mientras las plantas control mostraron un $32,6 \%$ de brotación, en los árboles defoliados brotó el 94,6\% de las yemas, lo cual reveló el beneficio de la remoción de las hojas luego de la cosecha, sobre la brotación de las yemas.

Palabras clave: Relación C/N, nitrógeno, carbono, peso seco.

\section{SUMMARY}

Although culture requirements have been established for 'Anna' apple trees grown in the tropical highlands, little is known about the physiological and biochemical events that mark the end of chilling requirements and the beginning of budbreak. Under tropical conditions in Colombia, floral buds of apple burst at any time of the year in response to removal of the leaves after harvest, to pruning and to spraying of chemical inducers. The present work was carried out to evaluate the response of apple trees cv. 'Anna' to defoliation timing before or after harvest time in Paipa, Colombia. Different trees were defoliated one week before, at harvest and one to four 
weeks after harvest. Control plants were not defoliated and leaves remained on twigs during the experiment time up to their natural fall. As response variables, nitrogen, carbon contents and the $\mathrm{C} / \mathrm{N}$ ratio in the floral buds at defoliation and at budbreak were measured; dry weight of the floral buds was also determined at defoliation and at budbreak, as well as the days from defoliation to burst. As results, the leaf removal reduced the time from defoliation to budbreak. The defoliation timing affected positively the burst percentage and the dry weight of floral buds, while nitrogen and carbon contents in buds remained unchanged. While control plants without leaf removal showed $32.6 \%$ budbreak, this was $94.6 \%$ for the trees defoliated one week after harvest, showing the beneficial effect of the defoliation on the budbreak.

Key words: C/N ratio, nitrogen, carbon, dry weight.

\section{INTRODUCCIÓN}

La variedad de manzano 'Anna' tiene un requerimiento aproximado de 300 horas frío y un requerimiento de 5.731 unidades acumuladas de calor (Growing degree hours) para plena floración, lo que es relativamente poco para una variedad de esta especie. Además, el período de desarrollo de los frutos es bastante corto (120 días) y es posible que esta variedad se pueda cultivar en los altiplanos tropicales colombianos desde 1700 a $2800 \mathrm{msnm}$. Estas características permiten la obtención de dos cosechas en un lapso de 12-14 meses, mediante la implementación de la técnica de cosechas continuas (Fischer E Lüdders, 1995; El-Agamy et al. 2001; Fischer et al. 2007). Este plan de manejo del cultivo comprende el seguimiento riguroso de las siguientes prácticas culturales: cosecha - estrés hídrico - defoliación - poda - riego - fertilización - aplicación de un compensador de frío - protección fitosanitaria - cosecha (Fischer, 1993; Casierra-Posada, 1993). Entre las prácticas de cultivo mencionadas, la defoliación y la poda son de gran importancia para incrementar el porcentaje de brotación de las yemas, lo cual redundaría en la reducción de la concentración del producto compensador de frío, como la cianamida hidrogenada (Díaz et al. 1987; Petri, 1992). Además, se utiliza la defoliación en fincas de pequeños agricultores, debido a los altos costos de los productos químicos empleados para el rompimiento de la dormancia (Fischer et al. 2007).

En la zona templada e incluso en la región subtropical, se defolian los árboles frutales de manera natural en la época de otoño; en los altiplanos tropicales, por el contrario, permanecen las hojas en los árboles por un periodo muy prolongado después de la cosecha, a raíz de que la diferencia en la duración del día a lo largo del año es muy escasa. Por esta razón, se deben retirar las hojas después de la cosecha para así incrementar la brotación de las yemas, para el inicio de una nueva temporada y poder lograr dos cosechas por año.

La defoliación, el arqueado y el despunte de las ramas son métodos comúnmente utilizados en el manejo de plantaciones de manzano en localidades diferentes a la zona templada. Entre ellos, la defoliación mostró una marcada diferencia en cuanto a la inducción de la brotación, respecto a los otros métodos, la cual tuvo lugar cuatro semanas luego de la aplicación del tratamiento. Se encontraron diferencias en cuanto al porcentaje de brotación cuando ésta se induce en época seca o lluviosa. Se concluyó que la defoliación es el estímulo más eficaz en la brotación inducida, si el suministro de agua no es limitante (Edwards \& Notodimedjo, 1987).

La defoliación, se puede realizar manualmente o mediante el uso de productos defoliantes concentrados, como urea, sulfato de cobre, cloruro de magnesio, cianamida hidrogenada (Erez, 1985; Díaz et al. 1987; Fischer $E$ Lüdders, 1995). Mediante la defoliación, se fomenta la brotación de las yemas reproductivas, dado que a través de ella se pueden retirar los inhibidores, como el ácido abscísico, presentes en las hojas (Edwards, 1987). El momento, en que se retiran las hojas, tiene una influencia directa sobre la brotación, pues la inducción floral se inhibe con una defoliación precoz (Fulford, 1970) o, por el contrario, se reduce la brotación cuando la defoliación se realiza demasiado tarde (Edwards, 1987). Mientras las yemas no hayan entrado en la fase de dormancia endógena, se inhibe su brotación, debido a los fitoreguladores del crecimiento contenidos en las hojas.

La relación C/N es un factor de gran relevancia en aquellas reacciones que tienen como resultado el crecimiento del vegetal; es así como el incremento en el suministro de nitrógeno en las plantas dirige una cantidad mayor de carbono hacia los aminoácidos y hacia la síntesis de proteínas y menos hacia los carbohidratos, por tanto, el contenido del azúcar y de almidón se reduce drásticamente en los tejidos de reserva, mientras que la cantidad total de carbohidratos estructurales sigue siendo generalmente constante (Drüge et al. 1998; Kaiser, 1997). 
Mediante la metodología utilizada para este ensayo, se pretendió determinar el momento más apropiado para la realización de la defoliación, con el fin de lograr el mayor porcentaje de brotación de yemas en un mínimo de tiempo. Por otro lado, se intentó establecer el efecto de la relación $\mathrm{C} / \mathrm{N}$ en las yemas reproductivas sobre la brotación, según la época de defoliación.

\section{MATERIALES Y MÉTODOS}

En la investigación, se utilizaron 21 árboles de siete años de edad de manzano 'Anna' ('Golden Delicious' x 'Adassim Red') sobre patrón MM-106, plantados en la granja 'Tunguavita' en Paipa - Boyacá, Colombia, ubicada a una altura de $2470 \mathrm{msnm}$. La localidad presenta $13,8^{\circ} \mathrm{C}$ de temperatura promedio anual y $74 \%$ de humedad relativa, con régimen bimodal de lluvias que aportan $835 \mathrm{~mm}$ de precipitación al año.

En cada uno de los árboles a tratar, se seleccionaron y se rotularon diez ramas del crecimiento de la última temporada, en las cuales se contaron la totalidad de las yemas, con el fin de determinar el porcentaje futuro de brotación. De estas ramas no se tomaron pruebas para análisis de tejidos.

En los árboles testigo (control), se tomó una muestra de cinco yemas reproductivas (lamburdas) de ramas que no habían sido rotuladas y que se encontraban en el tercio medio del árbol; en estas yemas, se determinó el peso fresco y seco y los contenidos de nitrógeno y carbono. La toma de las muestras en los árboles testigo, se realizó una vez por semana, partiendo una semana antes de la cosecha hasta la brotación de estos árboles.

La descripción de los tratamientos, se presenta en la tabla 1. Exceptuando los árboles control, en los manzanos en los que se realizaron los tratamientos, se tomaron muestras de yemas al momento de la defoliación y cuando sucedió la brotación; para la toma de las muestras, se siguió el mismo procedimiento que en los testigos, de tal manera que a partir de una semana antes de la cosecha hasta la sexta semana después de ésta, se defoliaron manualmente los árboles correspondientes a cada tratamiento y tanto el día de la defoliación como cuando se presentó la brotación, se tomaron muestras de las yemas reproductivas para determinar su contenido de carbono y de nitrógeno. También se realizaron observaciones periódicas para determinar la fecha de la brotación.

Análisis de muestras. El peso seco se determinó mediante el secado de las muestras en una mufla, a una temperatura de $65^{\circ} \mathrm{C}$ durante 24 horas. El porcentaje de peso seco, se obtuvo mediante la siguiente fórmula: $\mathrm{Tg}$ $(\%)=((\mathrm{Tg}-\mathrm{Fg}) / \mathrm{Tg}) \times 100$, en la cual $\mathrm{Tg}$ corresponde a peso seco y $\mathrm{Fg}$ a peso fresco. El contenido de nitrógeno en las yemas, se realizó con el método Kjeldahl, que consiste en la conversión de los compuestos nitrogenados reactivos a sulfato de amonio por mineralización de la muestra con ácido sulfúrico con un alto contenido de sulfato potásico, con objeto de elevar el punto de ebullición de la mezcla, en presencia de selenio, que actúa como catalizador (Instituto Geográfico 'Agustín Codazzi', 1973). Para determinar la concentración de carbono en las yemas, se utilizó el método Walkey-Black, que tiene como principio la oxidación de la materia orgánica con dicromato de potasio en medio ácido y posteriormente, una valoración del exceso de ácido crómico formado por retroceso con disolución de sal ferrosa, aprovechándose el calor de disolución del $\mathrm{H}_{2} \mathrm{SO}_{4}$ (Walkey y Black, 1934).

Análisis estadístico. Cada tratamiento se replicó tres veces y a la información obtenida en el ensayo, se le

Tabla 1. Descripción de tratamientos para la determinación del efecto de la época de defoliación sobre la brotación del manzano 'Anna'.

\begin{tabular}{|c|c|}
\hline DESCRIPTOR & TRATAMIENTO \\
\hline Control & Sin defoliar \\
\hline-1 & Defoliación una semana antes de la cosecha \\
\hline 0 & Defoliación al momento de la cosecha \\
\hline 1 & Defoliación una semana después de la cosecha \\
\hline 2 & Defoliación dos semana después de la cosecha \\
\hline 3 & Defoliación tres semana después de la cosecha \\
\hline 4 & Defoliación cuatro semana después de la cosecha \\
\hline
\end{tabular}


realizó un análisis de varianza (ANAVA, $\mathrm{P}<0,05)$ y la prueba de Duncan, con probabilidad de error de $5 \%$.

\section{RESULTADOS Y DISCUSIÓN}

El porcentaje de yemas brotadas mostró diferencias altamente significativas con respecto a la época de defoliación antes o después de la cosecha. Es así, como aquellas plantas que fueron defoliadas entre una y cuatro semanas después de la cosecha no mostraron diferencias significativas entre sí, pero sí hubo diferencias significativas $(\mathrm{P}<0,01)$ con los tratamientos aplicados una semana antes y al momento de la cosecha y con aquellas plantas que no se defoliaron (Figura 1).

De igual manera, otros investigadores han reportado que para lograr una mejor brotación de los árboles de manzano, en el trópico, se realiza la defoliación de manera manual o mediante agentes químicos, entre una a cuatro semanas después de la cosecha. Esta actividad representa una medida decisiva para evitar la entrada de las plantas en dormancia y, por tanto, como sucedió en el presente estudio, se logra una mayor brotación de las yemas (Edwards \& Notodimedjo, 1987; Fischer $\varepsilon$ Lüdders, 1995). De igual forma que en árboles de manzano, en plantas de chirimoyo, una manera de adelantar la emisión de flores, se basa en la defoliación del árbol, inducida mediante la aspersión con diferentes productos diluidos en agua después de la cosecha (George E Nissen, 1987; Magdahl, 1990; Razeto E Díaz, 2000). Bajo las condiciones cálidas de Australia es posible inducir una segunda defoliación y producción mediante la eliminación de las hojas lo cual genera brotes que emitirán flores cuatro semanas después. Esta práctica, se ha realizado con éxito en plantas de chirimoyo (Sanewski, 1991) y cumple las mismas funciones que la técnica de cosechas forzadas, reportada por Fischer $\mathcal{E}$ Lüdders (1995), que se lleva a cabo usualmente en Colombia, en cultivos tecnificados de manzano 'Anna'. Sin la práctica de la defoliación no sería posible la obtención de dos cosechas de manzana por año, dado que, como sucedió en el control (Figura 1), la brotación es escasa y sucede más tarde, como se discutirá más adelante.

La producción de manzano mediante la técnica de cosechas forzadas es posible en zonas tropicales y subtropicales, en donde se realiza la defoliación de los árboles luego de la cosecha (Janick, 1974; Notodimedjo et al. 1981). Mediante esta práctica, se previenen los cambios bioquímicos en las yemas, que culminan con el desarrollo de la dormancia en los meristemas. La época de la defoliación determina el momento de la brotación y, por tanto, de la cosecha, de manera que es posible una producción continua durante todo el año (Notodimedjo et al. 1981a). Esta práctica agrícola, se basa en que la remoción de las hojas maduras elimina las fuentes de ácido abscísico (ABA) y de otros inhibidores del crecimiento. Así, la reducción en el contenido de inhibidores permite que se incremente la concentración de giberelinas en las yemas, las cuales tienen una función promotora del crecimiento y. por tanto, la inducción a la brotación (Taylor et al. 1984). De hecho, la remoción de estructuras adyacentes al meristema resulta beneficioso en cuanto al incremento de la brotación. Se ha encontrado que el hecho de retirar parcialmente los catáfilos de las

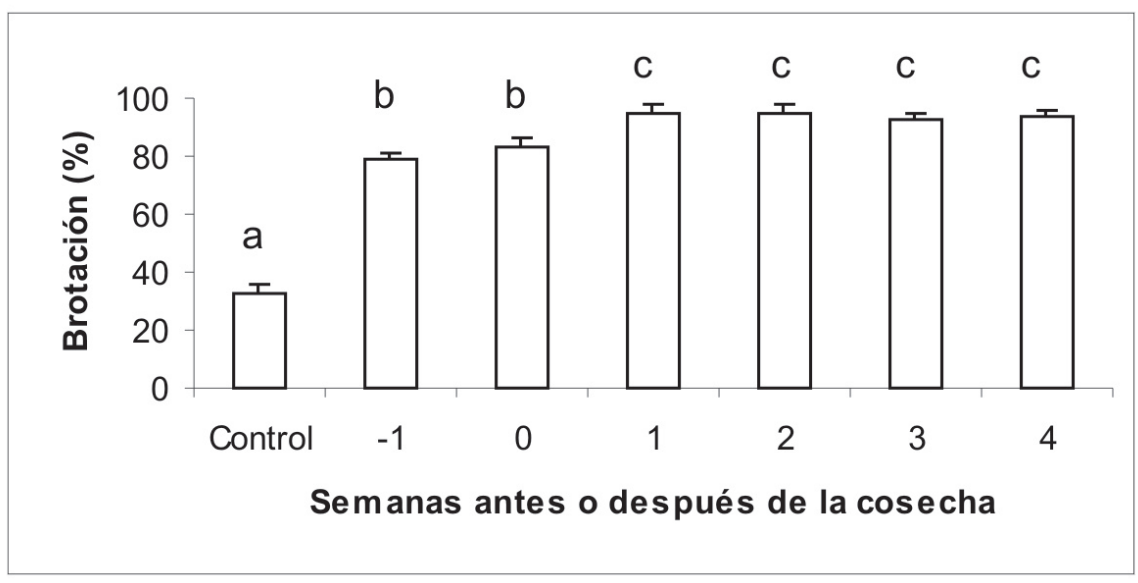

Figura 1. Porcentaje de brotación de las yemas del manzano sometido a diferentes épocas de defoliación. 
yemas en plantas de uva, lo cual produce un estrés por heridas es más efectivo en la etapa de paradormancia y endodormancia, que cuando se realiza este procedimiento en etapas posteriores (El-Shereif et al. 2006).

En cuanto a la variable peso seco en el momento de la defoliación, se encontró significancia al nivel de 0,05. En la figura 2 se observa que el peso seco disminuye hasta el momento de la cosecha y, posteriormente, la variable sufre un incremento hasta la segunda semana después de la cosecha. En cuanto al peso seco de las yemas registrado al momento de la brotación, se encontró diferencia altamente significativas $(P<0,01)$ y se observa que éste aumenta, en la medida que la defoliación se hace más tarde respecto al momento de la cosecha. La importancia de esta variable radica en que es un índice de la removilización de nutrientes y de la división celular, como consecuencia de la salida de las yemas de la dormancia.

De igual manera, Taylor et al. (1984) encontraron leves modificaciones en cuanto al peso seco de las yemas muestreadas en dependencia de la defoliación en árboles

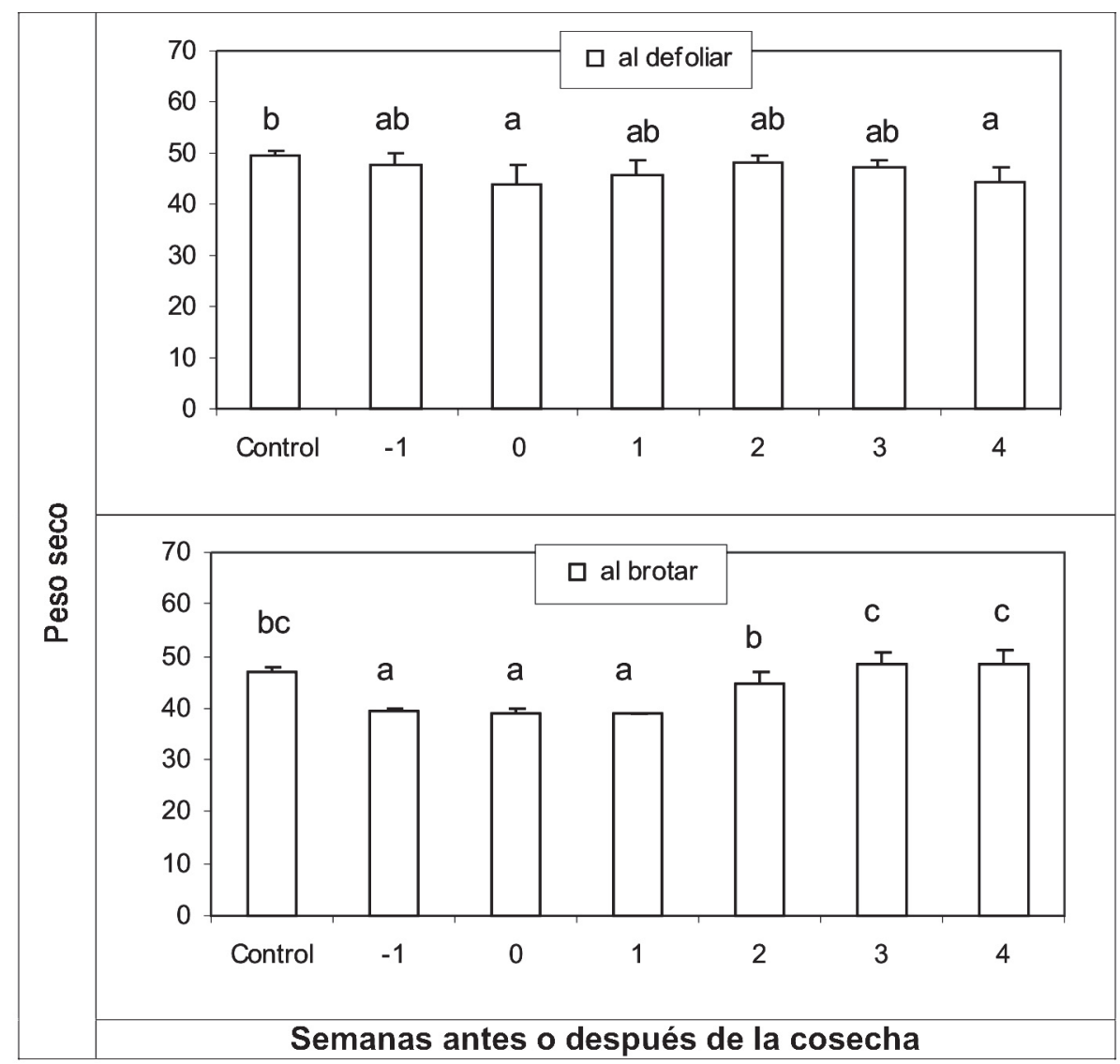

Figura 2. Peso seco en yemas del manzano 'Anna' sometidos a diferentes épocas de defoliación, determinado al momento de la defoliación y de la brotación de las yemas.

de manzano. Las variaciones en el peso seco pueden ser una consecuencia de la movilización y removilización de minerales y carbohidratos desde la corteza y las raíces hacia los órganos vertedero, representados por las yemas vegetativas y generativas en el momento de la brotación. Es así que los nutrientes y las fuentes de energía deben estar disponibles en los puntos de crecimiento, dado que la planta no dispone, en el momento, de otra fuente de recursos que favorezcan la brotación y el crecimiento de las yemas inducidas a través de la defoliación. Adicionalmente, en las yemas de manzano 'Anna', se presenta una diferenciación morfofisiológica que culmina con el desarrollo de los órganos florales 136 días después de plena floración, momento en que la yema reproductiva se encuentra completamente formada y lista para la brotación (Camargo, 1994). 
Adicionalmente, se ha encontrado que la realización de la defoliación en manzano, con dos semanas de diferencia, reduce la cantidad de ácido abscísico (ABA) e incrementó hasta tres veces la cantidad de giberalinas en las yemas, mientras el contenido de citoquininas permaneció casi inalterado. Estos cambios preceden tanto la apertura de las yemas como el incremento marcado en peso fresco y seco, los cuales son altamente dependientes del contenido y de balance de reguladores del crecimiento (Edwards, 1985).

Respecto a los días transcurridos entre la defoliación y el momento en que habían brotado el $50 \%$ de las yemas hubo diferencia altamente significativas $(P<0,01)$.
Además, se observó que en las plantas control, la brotación sucedió alrededor de 96 días después de iniciado el ensayo, mientras que en las plantas en donde se realizó defoliación, la brotación tuvo lugar entre los 35 y los 42 días después de la defoliación (Figura 3).

La disminución en el tiempo de brotación mediante la práctica de la defoliación, se puede explicar a través de la remoción de inhibidores producidos por las hojas con la práctica mencionada (Taylor et al. 1984), de manera que en las plantas control la brotación de las yemas fue más tardía que en las plantas defoliadas, dado que las hojas, como fuente de inhibidores, permanecieron más tiempo en la rama.

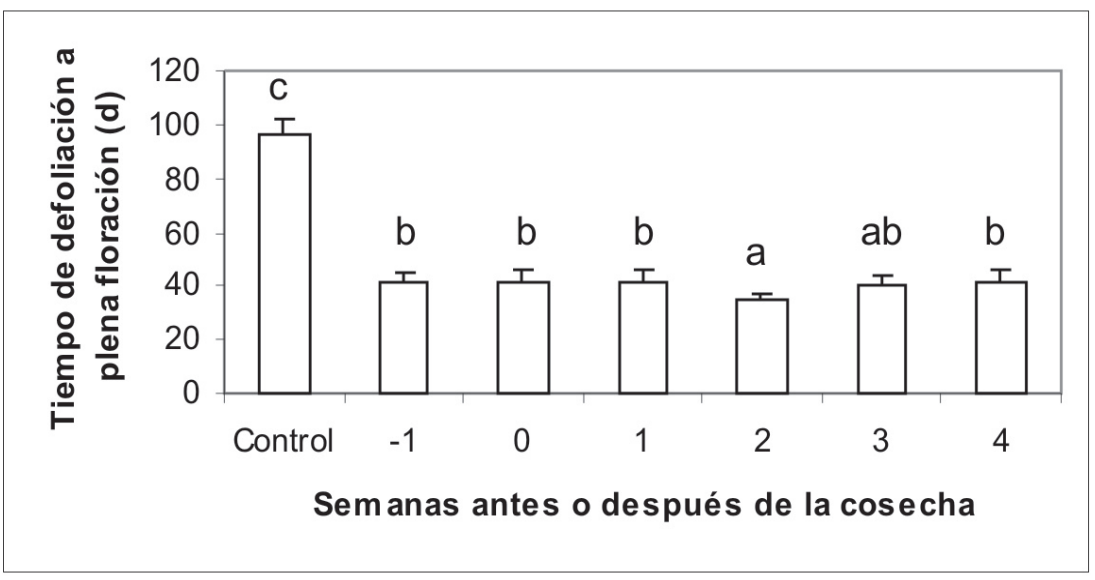

Figura 3. Tiempo transcurrido entre la defoliación y la plena floración en manzano 'Anna' sometido a diferentes épocas de defoliación.

De igual manera, la defoliación artificial realizada en árboles de cerezo previno el incremento en la concentración de ABA en los primordios foliares, normalmente asociada con la senescencia natural en las hojas (Mielke $\mathcal{E}$ Dennis, 1978). Por otra parte, el ABA endógeno no estaba implicada en la dormancia, como sí sucede con el ABA exógeno aplicado en inyección a ramas de manzano (Sterrett E Hipkins, 1980); por tanto, el retiro de las hojas, como fuente de inhibidores exógenos, contribuye a que se reduzca el lapso de defoliación a brotación, como reencontró el presente estudio.

Entre los porcentajes de nitrógeno y de carbono determinados al momento de la defoliación y al momento de la brotación, no se presentó diferencia estadísticamente significativa. A pesar de lo cual se ha encontrado en zonas templadas que el nitrógeno se moviliza en la época de primavera desde la corteza hasta los puntos en crecimiento. En un ensayo en el cual se realizó defoliación en manzano, se encontró en las lamburdas de plantas defoliadas un contenido más bajo de nitrógeno que en plantas sin defoliar. El nitrógeno total estuvo compuesto en partes casi iguales de nitrógeno proteico y de nitrógeno soluble (Faby \& Naumann, 1987). Por tanto, se considera que el contenido de nitrógeno tiene una dinámica dependiente de la fenología y está disponible en los momentos en que la planta no tiene las hojas, como órganos fuente, pero tiene una alta demanda del elemento, por parte de las yemas reproductivas, para la síntesis de proteínas y para el crecimiento.

Es posible que bajo condiciones tropicales, dado el manejo de cosechas forzadas, esa movilización y removilización del nitrógeno de las hojas a la corteza y viceversa reportada por Faby $\&$ Naumann (1987) no tenga lugar con una intensidad tan marcada, como sucede en zona templada. 
En esta última, el contenido de elementos nutritivos necesarios para el crecimiento en los primeros estadios de brotación de las plantas es altamente dependiente del fotoperiodo y del termoperiodo (Feucht, 1982; Friedrich E Fischer, 2000). En condiciones tropicales, estos factores no tendrían un cambio tan drástico, como sucede en zona templada, por tanto, en el trópico, los caducifolios se deben dejar guiar por los mecanismos orientados por sus relojes biológicos más que por los cambios en el fotoperiodo y en el termoperiodo.

Respecto a la movilización y removilización de carbohidratos es de anotar que la mayoría del carbono orgánico producido a través del proceso fotosintético lo utilizan numerosos órganos vertedero durante los periodos de crecimiento y de desarrollo. Luego del periodo de crecimiento, la exigencia de los órganos vertedero, se reduce y el almidón, que en su mayoría constituye la reserva de carbohidratos, se almacena durante el periodo en que el árbol está desprovisto de hojas como material de reserva para los procesos metabólicos que tendrán lugar al iniciar la próxima temporada de crecimiento.

Los patrones de acumulación de almidón y azúcares solubles en los tejidos reflejan el comportamiento fisiológico y la vitalidad de las plantas caducifolias desarrolladas en condiciones de clima templado; sin embargo, en la zona tropical, la implementación de la técnica de cosechas forzadas modifica parcialmente estos patrones de movilización de compuestos, dado que la defoliación puede llegar a limitar un poco la acumulación de compuestos en ramas y raíces, puesto que las plantas se defolian muy temprano y es posible que la movilización de los elementos presentes en las hojas no haya culminado aún. Las variaciones en los patrones de concentración de carbono en los tejidos reflejan cambios en el comportamiento fisiológico de las plantas y se pueden utilizar para la comprensión de la fisiología de los árboles que están siendo afectados por factores ambientales causantes de estrés (Parker $\mathcal{E}$ Houston, 1971; Parker, 1974; Wargo, 1981; Carroll et al. 1983; Gregory et al. 1986; Rasmussen E Henry, 1990; Renaud E Mauffette, 1991; Wong et al. 2001) o por condiciones de manejo agronómico, como la técnica de rest aviodance, reportada por Fischer $\mathcal{E}$ Lüdders (1995). En cuanto al metabolismo de los carbohidratos en árboles de manzano, se pueden tomar como referencia las plantas de peral, dado su parentesco. En estas plantas, se reporta que durante la fase que antecede la brotación, las reservas de almidón se transforman en azúcares solubles, lo cual puede ser un índice del final de la dormancia, pues en la medida en que las plantas completan el periodo de reposo, aumenta la demanda de azucares solubles para el reinicio del crecimiento; por tanto, la cantidad de almidón en las yemas puede ser un marcador de la superación de la dormancia. Además, la determinación de la superación de la dormancia es crucial para los productores, pues una brotación insuficiente o irregular compromete sustancialmente la producción. De igual manera, el conocimiento del metabolismo de los carbohidratos puede ser una referente importante para la determinación de la aplicación de productos o estrategias para la superación de la dormancia (Rodrígues et al. 2006)

Para entender mejor el efecto de la oferta ambiental sobre la distribución y el contenido de carbono en los tejidos es importante comprender los cambios en los niveles de carbohidratos relacionados con los eventos fenológicos y procesos fisiológicos durante el periodo fotosintético (desde el periodo de crecimiento activo hasta el almacenamiento de los carbohidratos) y el periodo no fotosintético (desde la dormancia hasta el inicio del crecimiento activo). Jones \& Bradlee (1933) determinaron que el contenido de almidón en árboles de Acer saccharum, determinado a la altura del pecho del observador, se reduce mensualmente desde noviembre a octubre. El muestreo de ramas permite la evaluación de los niveles de carbohidratos durante los periodos fotosintético y no fotosintético, información con la cual se puede entender el efecto del ambiente sobre la distribución de carbohidratos (Wong et al. 2003).

Con respecto al contenido de compuestos orgánicos en las yemas de árboles caducifolios, se reporta que el mayor porcentaje de carbohidratos solubles en relación con los insolubles en la entrada a la dormancia sugiere una mayor predisposición al transporte en la yema, mientras que el caso contrario en una dormancia más profunda sugiere una predisposición al almacenamiento para la siguiente brotación. El transporte pasivo de carbohidratos solubles puede tener lugar a pequeñas distancias mediante difusión simple o por transporte facilitado por proteínas transportadoras presentes en la membrana (Buckhout \& Tubbe, 1996; Raven et al. 2001; Carvalho $E$ Zanette, 2004). A pesar de estas apreciaciones, la implementación del manejo de plantas de manzano mediante cosechas continuas, modifica estos 
patrones convencionales de transporte, movilización y removilización de compuestos, como se encontró en el presente estudio, dado que la defoliación temprana implica alteraciones en el proceso de acumulación de minerales y de compuestos orgánicos.

Bajo condiciones de zona templada, se ha encontrado que en hojas de manzano, el nitrógeno removido en otoño puede ser menor que el nitrógeno removilizado en la primavera siguiente (Neilsen et al. 1997). Además, árboles con contenidos relativamente altos de nitrógeno en las hojas al inicio de la senescencia foliar, remueven a partir de ésta una proporción más baja del nitrógeno total de la hoja en comparación con aquellas que reciben un suministro moderado de nitrógeno (Tagliavini et al. 1998; Rosecrance et al. 1998; Tagliavini E Millard, 2005). Esto implica un patrón común en caducifolios; sin embargo, en árboles caducifolios que crecen en zona tropical estos patrones se alteran dado que las plantas no disponen de cambios drásticos en el fotoperiodo y termoperiodo, como ya se comentó.

\section{CONCLUSIONES}

La defoliación se convierte en una práctica obligada para el manejo de árboles de manzano bajo la técnica de cosechas continuas, dado que a través de ella es posible mantener una brotación uniforme y abundante; sin embargo, el momento en que se realice la defoliación tiene un papel determinante tanto en el momento en que suceda la brotación como en el porcentaje de yemas brotadas. Con los resultados del presente estudio es posible asumir que dos semanas después de la cosecha es una época adecuada para la realización de la defoliación, dado que se logra una adecuada brotación y se le da tiempo a que suceda la movilización de minerales y compuestos orgánicos para la siguiente brotación.

\section{AGRADECIMIENTOS}

Los autores agradecen al personal de la granja 'Tunguavita' de la Universidad Pedagógica y Tecnológica de Colombia por su colaboración en la realización del presente trabajo. Este estudio fue desarrollado con el apoyo de la Dirección de Investigaciones (DIN) de la Universidad Pedagógica y Tecnológica de Colombia, en el marco del plan de trabajo del grupo de investigación Ecofisiología Vegetal, adscrito al programa de Ingeniería Agronómica de la Facultad de Ciencias Agropecuarias.

\section{BIBLIOGRAFÍA}

BUCKHOUT, T.J.; TUBBE, A. 1996. Structure, mechanisms of catalysis, and regulation of sugar transporters in plants. En: Zamski, E.; Schaffer, A.A. eds. Photoassimilate distribution in plants and crops: source-sink relationships. Ed. Marcel Dekker (New York). p.229-260.

CAMARGO S., J. 1994. Determinación de la época de inducción floral en manzano 'Anna' (Malus domestica B.) en condiciones tropicales. Trabajo de grado de Ingeniero Agrónomo. UPTC, Tunja. 94p.

CARROLL, J.E.; TATTAR, T.A.; WARGO, P.M. 1983. Relationship of root starch to decline of sugar maple. Plant Dis. 67:1347-1349.

CARVALHO, R.I.N.; ZANETTE, F. 2004. Conteúdo de carboidratos em gemas e ramos de macieira durante o outono e inverno em região de baixa ocorrência de frio. Rev. Bras Frutic. Jaboticabal. 26(2):202-205.

CASIERRA-POSADA, F. 1993. Protección y nutrición para cosechas continuas en manzano 'Anna'. Agrodesarrollo. 4(1-2):46-49.

DÍAZ, D.; ÁlVAREZ, A; SANDOVAL, J. 1987. Cultural and chemical practices to induce uniform bud break of peach and apple under warm climates. Acta Hort. 199:129-136.

DRÜGE, U.; ZERCHE, S.; KADNER, R. 1998. Relation between nitrogen and soluble carbohydrate concentrations and subsequent rooting of chrysanthemum cuttings. Advances in Hort. Sci. 12:78-84.

EDWARDS, G.R. 1985.Changes in endogenous hormones in apple during bud burst induced by defoliation. Acta Hort. 158: 203-210.

EDWARDS, G.R. 1987. Producing temperate zone fruit at low latitudes: avoiding rest and chilling requirement. HortScience 22(6):1236-1240.

EDWARDS, G.R.; NOTODIMEDJO, S. 1987. Defoliation, bending and tip pruning of apple under tropical conditions. Acta Hort. 199:125-127. 
EL-AGAMY, S.Z.; MOHAMED, A.K.A.; MOSTAFA, F.M.A.; ABDALLAH, A.Y. 2001. Chilling and heat requirements for budbreak and fruiting of "Anna" and "Dorsett Golden" apple cultivars under warm climatic conditions. Acta Hort. 565: 103-108.

El-Shereif, A.R.; Mizutani, F.; Onguso, J.M.; Sharif Hossain, A.B.M. 2006. Effect of bud scale removal and AOA on bud break and ACC content of 'Muscat Bailey A' grapevines. J. Appl.Hort. 8(2):125-128.

EREZ, A. 1985. Defoliation of deciduous fruit trees with magnesium chlorate and cyanamide. HortScience. 20(3):452-453.

FABY, R.; NAUMANN, W.D. 1987 Die Bedeutung der Einlagerung von Reservestoffen im Herbst bei Apfelbäumen, dargestellt an Entblätterungsversuchen. Erwerbstobstbau. 29:51-56

FEUCHT, W. 1982. Das Obstgehölz. Ulmer Verlag. Stuttgart. p.114-168.

FISCHER, G. 1993. Fisiología en la producción de dos cosechas anuales en manzano y durazno. Agrodesarrollo. 4(1-2):18-31.

FISCHER, G.; LÜDDERS, P. 1995. Der Apfelanbau im Hochland Kolumbiens. Erwerbsobstbau. 37:58-62.

FISCHER, G.; LASPRILLA, D.M.; CASIERRA-POSADA, F. 2007. Current state of temperate fruits in Colombia (South America). En: VIII Internl. Symp. of temperate zone fruits in the tropic and subtropic,. Riesser Júnior, C.; Simões, F.; Souza Zonato, W.; Rodrigues Corrêa, E. (Eds.). Embrapa. Florianopolis - Santa Catarina, Brazil. p.107.

FRIEDRICH, G.; FISCHER, M. 2000. Regelmechanismen der Gehölzentwicklung. En. Physiologische Grundlagen des Obstbaues. Ulmer Verlag. Stuttgart. p.200-209.

FULFORD, R.M. 1970. Leaves, fruit and flower initiation. Proc. $18^{\text {th }}$ Int. Hort. Congr. 4:143-150.

GEORGE, A.; NISSEN, R. 1987. Effects of cincturing, defoliation and summer pruning on vegetative growth and flowering of custard apples in subtropical Queensland. Aust. J. Exp. Agric. 27:915-918.
GREGORY, R.A.; WILLIAMS, J.W.Jr.; WONG, B.L.; HAWLEY, G.J. 1986. Proposed scenario for dieback and decline of Acer saccharum in northeastern U.S.A. and southeastern Canada. IAWA Bull. New Ser. 7:357-369.

INSTITUTO GEOGRÁFICO 'AGUSTÍN CODAZZI'. 1973. Métodos analíticos del laboratorio de suelos. Bogotá D.C. p. 6-8

JANICK, J. 1974. The apple in Java. HortScience. 9:1315.

JONES, C.H.; BRADLEE, J.L. 1933. The carbohydrate contents of the maple tree. Bull. 358. Vermont Experiment Station, Burlington. p.1-147.

KAISER, W.M. 1997. Regulatory interaction of carbon and nitrogen metabolism. En: Behnke, H.D.; Lüttge, U.; Esser, K.; Kadereit, J.W. Runge, M. eds. Progress in Botany 58:150-163.

MAGDAHL, C. 1990. Efecto de la defoliación anticipada sobre la brotación, floración y desarrollo de frutos en chirimoyo cv. Concha Lisa y efectividad de algunos productos como defoliantes. Trabajo de grado, Fac. Agronomía. Quillota, Chile. U. Católica de Valparaíso. 92p.

MIELKE, E.A.; DENNIS, F.G. 1978. Hormone control of flower bud dormancy in sour cherry (Prunus cerasus L.). III. Effect of leaves, defoliation and temperature on levels of abscisic acid in flower primordial. J. Amer. Hort. Sci. 103:446-449.

NEILSEN, D.; MILLARD, P.; NEILSEN, G.H.; HOGUE, E.J. 1997. Sources of $N$ used for leaf growth in a high density apple (Malus domestica) orchard irrigated with ammonium nitrate solution. Tree Physiol. 17:733-739.

NOTODIMEDJO, S.; DANOESASTRO, H.; SASTROSUMARTO, S.; EDWARDS, G.R. 1981. Shoot growth, flower initiation and dormancy of apple in the tropics. Acta Hort.120:179-186.

NOTODIMEDJO, S.; DANOESASTRO, H.; SASTROSUMARTO, S.; EDWARDS, G.R. 1981a. Growth periodicity of apples under tropical conditions. Acta Hort. 120:256-256. 
PARKER, J. 1974. Effects of defoliation, girdling, and severing of sugar maple trees on root starch sugar levels. U.S. For. Serv. Res. Pap. NE-306.

PARKER, J.; HOUSTON, D.R. 1971. Effects of repeated defoliation on root and root collar extractives of sugar maple trees. Forest. Sci. 17:91-95.

PETRI, J. L. 1992. Superación del reposo de manzano en los altiplanos tropicales. Acta Hort. 310:15-22.

RAVEN, P. H.; EVERT, R. F.; EICHHORN, S.E. 2001. Biología vegetal. Río de Janeiro: Ed. Guanabara Koogan. 906p.

RASMUSSEN, T.S.; HENRY, R.J. 1990. Starch determination in horticultural plant material by an enzymic-colorimetric procedure. J. Sci. Food Agric. 52:159-170.

RAZETO, B.; DÍAZ, E. 2000. Producción forzada mediante deshoje y recorte de brotes en chirimoyo (Annona cherimola Mill.). Agr. Téc. 60(2):173-177.

RENAUD, J.P.; MAUFFETTE, Y. 1991. The relationships of crown dieback with carbohydrate content and growth of sugar maple (Acer saccharum). Can. J. For. Res. 21:1111-1118.

RODRÍGUES, A.C.; VERISSIMO, V.; CHAVARRIA, G.; CAMELATTO, D.; HERTER, F.G. 2006. Método para estimar o conteúdo de amido em gemas florais de pereira antes da brotação. Rev. Bras. Agroc.12(4):499-502.

ROSECRANCE, R.C.; JOHNSON, R.S.; WEINBAUM, S.A.. 1998. The effect of timing of post-harvest foliar urea sprays on nitrogen absorption and partitioning in peach and nectarine trees. J. Hort. Sci. 73(6):856-861

SANEWSKI, G. 1991. Custard apples, cultivation and crop protection. Queensland Dept. of Primary Industries. Brisbane, Australia. 103p.
STERRETT, J.P.; HIPKINS, P.L. 1980. Responses of apple buds to pressure injection of abscisic acid and cytokinin. J. Am. Soc. Hort. Sci. 105:917-920.

TAGLIAVINI, M.; MILLARD, P. 2005. Fluxes of nitrogen within deciduous fruit trees. Acta Sci. Pol., Hortorum Cultus. 4(1):21-30.

TAGLIAVINI, M.; MILLARD, P.; QUARTIERI, M. 1998. Storage of foliar-absorbed nitrogen and remobilization for spring growth in young nectarine (Prunus persica var. nectarina) trees. Tree Physiol. 18:203-207.

TAYLOR, J.S.; PHARIS R., P.; LOVEYS, B.; NOTODIMEDJO, S.; EDWARDS, G.R. 1984. Changes in endogenous hormones in apple during bud burst induced by defoliation. Plant Growth Regul. 2:117-134.

WARGO, P.M. 1981. Defoliation and tree growth. En: Doane, C.C.; McManus, M.L. (eds.) The gypsy moth: research towards integrated pest management. U.S. For. Serv. Tech. Bull. 1384. p.225-240.

WALKLEY, A.; BLACK, T.A. 1934. An examination of the Degtjareff methods for determining of soil organic matter, and a proposed modification of the chromic acid titration method. Soil Sci. 37:29-38.

WONG, B.L.; BAGGETT, K.L.; BURFEIND, A.S.; RYE, A.H. 2001. Carbohydrate profiles in woody tissues of sugar maples with crown dieback symptoms during the leafless period. En: L'Arbre 2000 - 2000 The Tree: Proc. Fourth Internal. Symposium on the Tree, Montreal, Que., 20-25 August 2000. M. Labrecque ed. IQ Collectif,. p.314-319.

WONG, B.L.; BAGGETT, K.L.; RYE, A.H. 2003. Seasonal patterns of reserve and soluble carbohydrates in mature sugar maple (Acer saccharum). Can. J. Bot. 81(8):780-788.

Recibido: Diciembre 9 de 2007

Aceptado: Abril 15 de 2008 